\title{
Análise térmica bidimensional de perfil de aço sujeita a elevadas temperaturas
}

\author{
Neves N. S*1; Azevedo, M. S. ${ }^{1}$; Camargo R. S. ${ }^{1}$, Pinheiro, V. P. ${ }^{2}$ \\ 1 Programa de Pós-Graduação em Engenharia Civil, Universidade Federal do Espírito Santo, Vitória, ES, Brasil. \\ 2 Programa de Pós-Graduação em Engenharia Mecânica, Universidade Federal do Espírito Santo, Vitória, ES, Brasil.
}

* e-mail: natan.sian@gmail.com

\section{Resumo}

O presente artigo objetiva estudar o comportamento térmico em um perfil $\mathrm{H}$ de aço com e sem proteção térmica tipo contorno de espessura uniforme via método de elementos finitos. Desta forma, aplica-se os procedimentos baseados no método de resíduos ponderados de Galerkin. A experimentação numérica é conduzida pelo comportamento da temperatura no centro da alma para níveis diferentes de tempo de exposição ao incêndio e por meio da análise paramétrica da espessura do material de proteção. Em síntese, o programa desenvolvido empregado para solução apresentou um bom ajuste em relação as respostas numéricas obtidas na literatura.

Palavras chaves: Análise Térmica; Aço; Elementos Finitos; Condição de Incêndio.

\begin{abstract}
The present article aims to study the thermal behavior in a $\mathrm{H}$ profile of steel with and without boundary thermal protection of uniform thickness by finite element method. In this way, the procedures based on the Galerkin weighted residue method are applied. Numerical experimentation is conducted by the behavior of the temperature in the center of the web for different levels of time of exposure to fire and by parametric analysis of the thickness of the protective material. In summary, the program developed for solution presented a good adjustment in relation to the numerical answers obtained in the literature.
\end{abstract}

Keywords: Thermal Analysis; Steel; Finite elements; Fire condition.

\section{Introdução}

Devido as diversas ocorrências envolvendo incêndio, houve uma crescente demanda de pesquisa científica acerca de analisar o comportamento de estruturas neste tipo de condição. Dentre os direcionamentos, a predição de temperatura em elementos estruturais é um dos importantes focos de aplicação da área.

Em projetos de estruturas de aço em situação de incêndio, pode-se mencionar a EN 19931-2:2005 [1]. Esta norma fornece métodos de cálculos simplificados para análise térmica em elementos de aço, possibilitando ainda, o uso de métodos avançados baseados nas hipóteses e princípios da transferência de calor, sendo usualmente apoiadas por técnicas numéricas de solução, como o método de elementos finitos .

\section{Formulação de Elementos Finitos}

$\mathrm{Na}$ análise da condução de calor, um dos pontos principais é determinar o campo escalar de temperatura em um meio com as devidas condições externas impostas ao sistema. Deste modo, deve-se obter um modelo matemático capaz de descrever tal fenômeno, para isso, aplica-se o princípio da conservação de energia em um volume de controle diferencial para obter a equação da difusão de calor [2], expressa na Equação (1).

$$
\nabla^{T} \mathbf{D} \nabla T+\dot{Q}-\rho c \frac{\partial T}{\partial t}=0
$$

Onde $T$ é a temperatura, $\dot{Q}$ é a geração de energia interna por unidade de volume, $\rho$ é 
a massa específica, $c$ é o calor específico, $\mathbf{D}=k \mathbf{I}$ é conhecida como matriz de condutividade térmica, para um material isotrópico. Em condições de incêndio, a troca de calor ocorre por convecção e radiação, a Equação (2) apresenta o fluxo no contorno.

$$
\begin{aligned}
& q_{n}=\alpha_{c}\left(T-T_{g}\right)+\alpha_{r}\left(T-T_{g}\right) \\
& \alpha_{r}=\varepsilon \sigma\left(T-T_{g}\right)\left(T^{2}-T_{g}{ }^{2}\right)
\end{aligned}
$$

Onde $T_{g}$ é a temperatura dos gases quentes em torno da estrutura, $\alpha_{c}$ e $\alpha_{r}$ são os coeficientes de convecção e radiação, $\sigma$ é a constante de Stefan-Boltzmann e $\varepsilon$ é a emissividade. Observe que radiação ao longo do tempo de exposição torna-se predominante, devido o fluxo ser elevada a quarta potência. Uma vez exposta a equação de governo do problema, os procedimentos de elementos finitos iniciam-se escrevendo a Equação (1) como uma sentença integral conjunta com uma função auxiliar $w$, conforme expresso na Equação (3).

$$
\int_{\Omega}\left(\nabla^{T} \mathbf{D} \nabla T+Q-\rho c \frac{\partial T}{\partial t}\right) w d \Omega=0
$$

Dentre as características da função peso, vale destacar que tal função deve ser nula no todo contorno essencial [2]. Aplicando a integração por partes na Equação (3) e introduzindo as condições de contorno expressa na Equação (2), chega-se na Equação (4).

$$
\begin{aligned}
& \int_{\Omega} \nabla^{T} w(\mathrm{D} \nabla T) d \Omega+\int_{\Omega} \rho c \frac{\partial T}{\partial t} w d \Omega \\
& -\int_{\Omega} Q w d \Omega+\int_{\Gamma} w q_{n} d \Gamma=0
\end{aligned}
$$

A Equação (4) é conhecida como formulação variacional fraca do problema de difusão de calor transiente com geração interna. Usando as funções de forma, pode-se reescrever os campos aproximados de temperatura e da função peso.

$$
T=\sum_{i=1}^{n} N_{i} \tilde{T}_{i} \quad w=\sum_{j=1}^{n} N_{j} \tilde{w}_{j}
$$

Sendo $N$ as funções de forma. Então, a formulação variacional discreta é gerada quando aplica-se as aproximações apresentada na Equação (5) na formulação fraca do problema, expressa pela Equação (4). Reorganizando, é possível escrever o sistema linear na forma compacta matricial, conforme Equação (6).

$$
\mathbf{C} \frac{\partial \tilde{T}}{\partial t}+\mathbf{K} \tilde{T}=\mathbf{F}
$$

As matrizes constituídas no sistema final são apresentas na Equação (7). Sendo que o vetor gradiente pode ser escrito em conjunto com a função de interpolação de elementos finitos $\nabla T=\nabla \mathrm{N} \tilde{T}=\mathbf{B} \tilde{T}$.

$$
\begin{aligned}
& \mathrm{K}=\int_{\Omega} \mathbf{B}^{\mathrm{T}} \mathbf{D} \mathbf{B} d \Omega+\int_{\Gamma} \mathbf{N}^{\mathrm{T}} \mathbf{N} \alpha d \Gamma \\
& \mathbf{C}=\int_{\Omega} \mathbf{N}^{\mathrm{T}} \rho c \mathbf{N} d \Omega \quad \mathbf{F}=\int_{\Gamma} \mathbf{N}^{\mathrm{T}} \alpha T_{g} d \Gamma
\end{aligned}
$$

O coeficiente $\alpha$ é definido como a soma do coeficiente de convecção e radiação. O tratamento da parte temporal é direcionado a partir do método de integração direta, assumindo a variação linear ao longo do passo de tempo, chega-se no expressão para a temperatura no passo $t_{n+1}$.

$$
\begin{aligned}
& \widehat{\mathbf{A}}=\tilde{T}_{n+1} \widehat{\mathbf{B}} \\
& \widehat{\mathbf{A}}=\left(\frac{\mathbf{C}}{\Delta t}+\theta \mathbf{K}\right) \\
& \widehat{\mathbf{B}}=\left(\frac{\mathbf{C}}{\Delta t}-(1-\theta) \mathbf{K}\right) \tilde{T}_{n}+(1-\theta) \mathbf{F}_{n}+\theta \mathbf{F}_{n+1}
\end{aligned}
$$

O parâmetro $\theta$ varia de 0 a 1 , sendo considerado no trabalho o método de Galerkin, o valor é igual a $2 / 3$ [2].

\section{Estudo de Caso}

Considere um perfil $\mathrm{H}$ de aço sujeito a curva de incêndio padrão ISO 834:1999 [3], a proteção térmica é constituída por uma argamassa projetada Blaze Shield II, conforme ilustra Figura 1. 


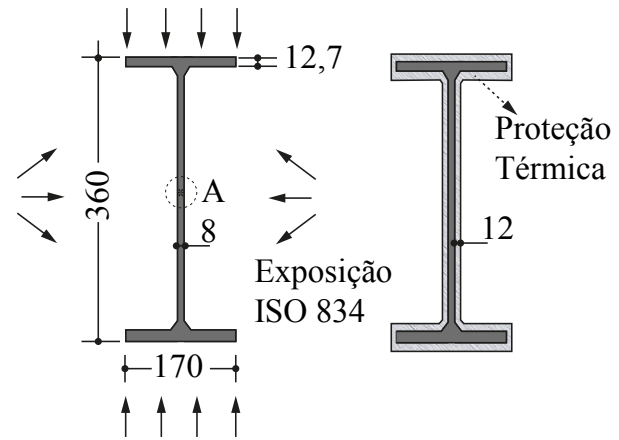

Figura 1: Dimensões do Perfil de aço sem e com proteção térmica

A validação dos resultados obtidos no presente trabalho são conduzidos pela comparação com o programa SAFIR [4]. A Figura 2 apresenta o perfil de temperatura versus tempo para o ponto A no centro da alma.

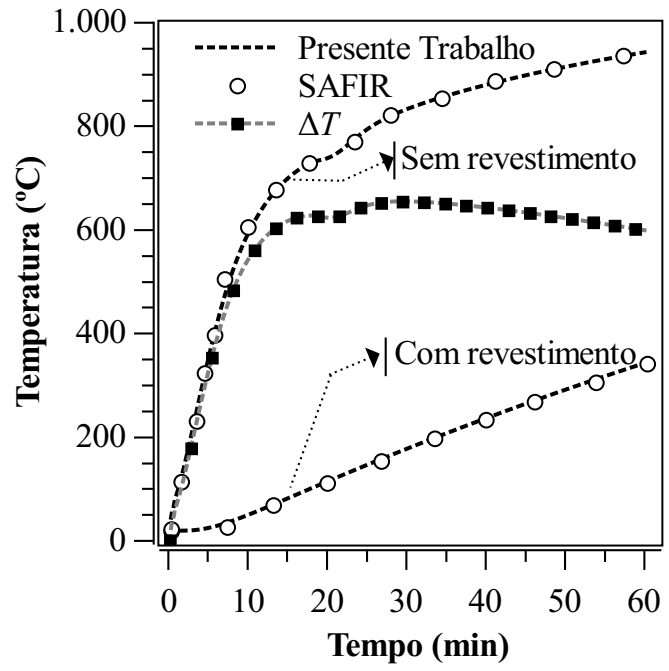

Figura 2: Variação de temperatura no ponto A

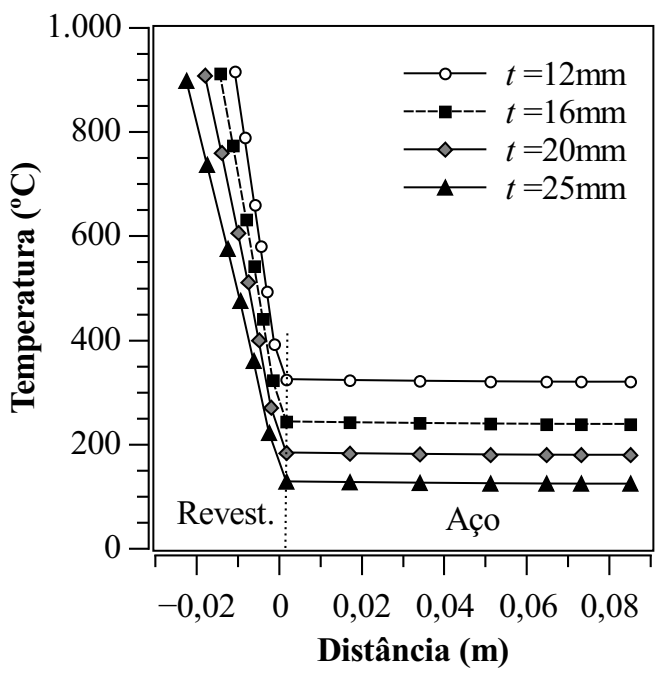

Figura 3: Perfil de temperatura na mesa inferior do perfil $\mathrm{H}$ de aço

Nota-se que a existe uma diferença signifi- cativa entre as curvas de temperatura para o perfil sem ou com proteção térmica. A variação de temperatura $\Delta T$ entre as curvas evidência que após aproximadamente $15 \mathrm{~min}$, a diferença fica um pouco acima de $600^{\circ} \mathrm{C}$. Esta ordem de grandeza entre as medições apresenta uma influência considerável para dimensionamento de estruturas de aço contra incêndio. Na Figura 3 é possível observar que quanto maior a espessura da proteção, menor os níveis de temperatura dentro do perfil. Nota-se também que a temperatura no aço mantém-se aproximadamente constante ao longo do perfil.

\section{Conclusão}

O programa desenvolvido para análise térmica não linear de estruturas sob condição de incêndio apresentou bons resultados quando comparados com a solução de referência. $\mathrm{O}$ estudo evidência a diferença significativa nos resultados obtidos para temperatura quando utiliza-se a proteção térmica, que seu uso torna-se imprescindível em perfis de aço.

\section{Referências}

[1] EN 1993-1-2. Eurocode 3: Design of steel structures - part 1-2: General rules - structural fire design. European Standards, London, CEN, Brussels, Belgium, 2005.

[2] REDDY, J. N. An introduction to the finite element method. New York: McGraw-hill, 1993. v. 2

[3] ISO 834. Fire resistance tests-elements of building construction. International Organization for Standardization, Geneva, Switzerland, Revision of frst edition ISO 834:1975, 1999.

[4] PIRES, D. et al. Análise térmica de seções transversais via método dos elementos finitos. In: XXXVI Congresso Ibero-Latino-Americano de Métodos Computacionais em Engenharia. RJ, Brasil, v.1 , p. 1-19, 2015 